\title{
Textile Dye Removal Using Photocatalytic Cascade Disk Reactor Coated by ZnO Nanoparticles
}

\author{
Hamid Amiri, Bita Ayati, Hossein Ganjidoust \\ Faculty of Civil and Environmental Engineering, Tarbiat Modares University, Tehran, Iran \\ Email: H-GANJI@Modares.ac.ir
}

How to cite this paper: Amiri, H., Ayati, B. and Ganjidoust, H. (2016) Textile Dye Removal Using Photocatalytic Cascade Disk Reactor Coated by ZnO Nanoparticles. Journal of Materials Science and Chemical Engineering, 4, 29-38.

http://dx.doi.org/10.4236/msce.2016.412004

Received: November 18, 2016

Accepted: November 25, 2016

Published: December 1, 2016

\begin{abstract}
Dye effluents with low BOD/COD ratio and varied chemical structures are usually very recalcitrant to microbial degradation. Therefore, different process was used for the treatment of dye effluent. Heterogeneous photocatalyst process has been widely used as leading green technology for dye removal. The process utilizes a semiconductor photocatalyst (such as $\mathrm{TiO}_{2}$ or $\mathrm{ZnO}$ ) and UV light to oxidize the recalcitrant organic compounds to inorganic ions, carbon dioxide and water. Photocatalytic process needs to photorectors and hydraulic parameters play an important role in mass transfer phenomenon in photocatalytic reactors. These fundamental parameters are flow rate, relative roughness and Reynolds number. This research experimentally evaluates flow rate and artificial relative roughness in order to determine the factors influencing the removal efficiency and reaction rate. For this purpose, a cascade photocatalytic reactor is constructed which consists of similar Plexiglas plates coated by various roughness. Numerical simulation usually overcomes complex reactor models which takes reasonable cost respect to experimental study. Here, OpenFOAM software is also utilized to perform a numerical study. Regime and velocity of sewage are simulated in photocatalytic flow with/without considering relative roughness.
\end{abstract}

\section{Keywords}

Cascade Disc Reactor, M5P, Reactive Yellow 81, Zinc Oxide, Artificial Roughness

\section{Introduction}

The use of different synthetic dyes in textile industries has increased in recent decay, resulting in the release of dye-containing industrial effluents into natural aquatic ecosystem [1]. Dye effluents with low BOD/COD ratio and varied chemical structures are usually very recalcitrant to microbial degradation. Therefore, dye removal from effluent 
is a main concern in many studies. Different process was used for the treatment of dye effluent. In the last few years, studies were focused on advanced oxidation process (AOPs) methods such as $\mathrm{UV}-\mathrm{ZnO}, \mathrm{UV}-\mathrm{H}_{2} \mathrm{O}_{2}, \mathrm{UV}-\mathrm{O}_{3}$ and $\mathrm{UV}-\mathrm{TiO}_{2}$ [2] [3]. Photocatalytic process such as $\mathrm{UV}-\mathrm{ZnO}$ is an efficient method that treats nondegredable wastewater by active radicals. The photocatalysis needs a photoreactor that contacts reactant, products and light. In recent years, different types of photoreactors have been used for wastewater treatment. In some reactors, nano-photocatalysts are utilized in slurry form, and the other particles are coated on bed. In Photocatalytic reactors with fixed bed, nano-photocatalysts are immobilized on bed and do not need the separation unit, but the main disadvantage of this photoreactors is the low mass transfer rate between wastewater and nano-photocatalysts [4]. Consequently, Different optimal photoreactors were developed for increasing mass transfer rate. Dionysiou et al. investigated the effect of rotational speed on 4-cholorophenol degradation using rotating disk photocatalytic reactor. They concluded that the rate of 4-cholorophenol degradation increased with rotating speed change from 5 to $20 \mathrm{rpm}$. Similarity, Son et al. used rotating drum photocatalytic reactor for Bisphenol degradation. The treatment efficiency of 97 and $99 \%$ were reported in the rotational speed of 60 and $120 \mathrm{rpm}$, respectively. Vezzoli, et al. studied the effect of flow rate on mass transfer rate in photocatalytic reactor with immobilized bed in which Reynolds number is limited to the domain of 350 - 3050, and therefore they revealed that flow rate increasing and large Reynolds number lead to increasing of mass transfer rate in a photocatalytic process [5]. Therefore, there are several ways for improving mass transfer of the photocatalytic reactors. The aforementioned literature review emphasizes that the studies had focused on mechanical methods such as rotational speed, agitation speed and flow rate for increasing mass transfer rate in photocatalytic process. In this study, in order to overcoming mass transfer limitation in photocatalytic process, a novel photocatalytic cascade disc reactor coated with $\mathrm{ZnO}$ nano-photocatalysts was applied and artificial roughness were created on the surface of disks.

This photoreactor has a number of advantages that include eliminating the need for catalyst separation units as the catalyst is immobilized, creating the flow mixing by non-mechanical method, increasing the transport of oxygen from the gas phase to the photocatalyst surface by providing the flow cascade pattern. The photoreactor was used in order to remove Reactive Yellow 81 (RY81) dye from textile industry effluent, by means of UV-ZnO process. RY81 is a reactive dye composed of 10 Benzene rings and two $-\mathrm{N}=\mathrm{N}$ azo bonds. The effect of different artificial roughness and recirculation flow rate in removal efficiency was investigated.

\section{Applied Materials and Methods}

\subsection{Cascade Disc Reactor Setup}

In order to remove the RY81 dye, a photocatalytic cascade disc reactor is constructed, which consists of four $34 \mathrm{~cm}$ diameter circular disc made of Plexiglas and immobilized by $\mathrm{ZnO}$ nanoparticles (Figure 1). 

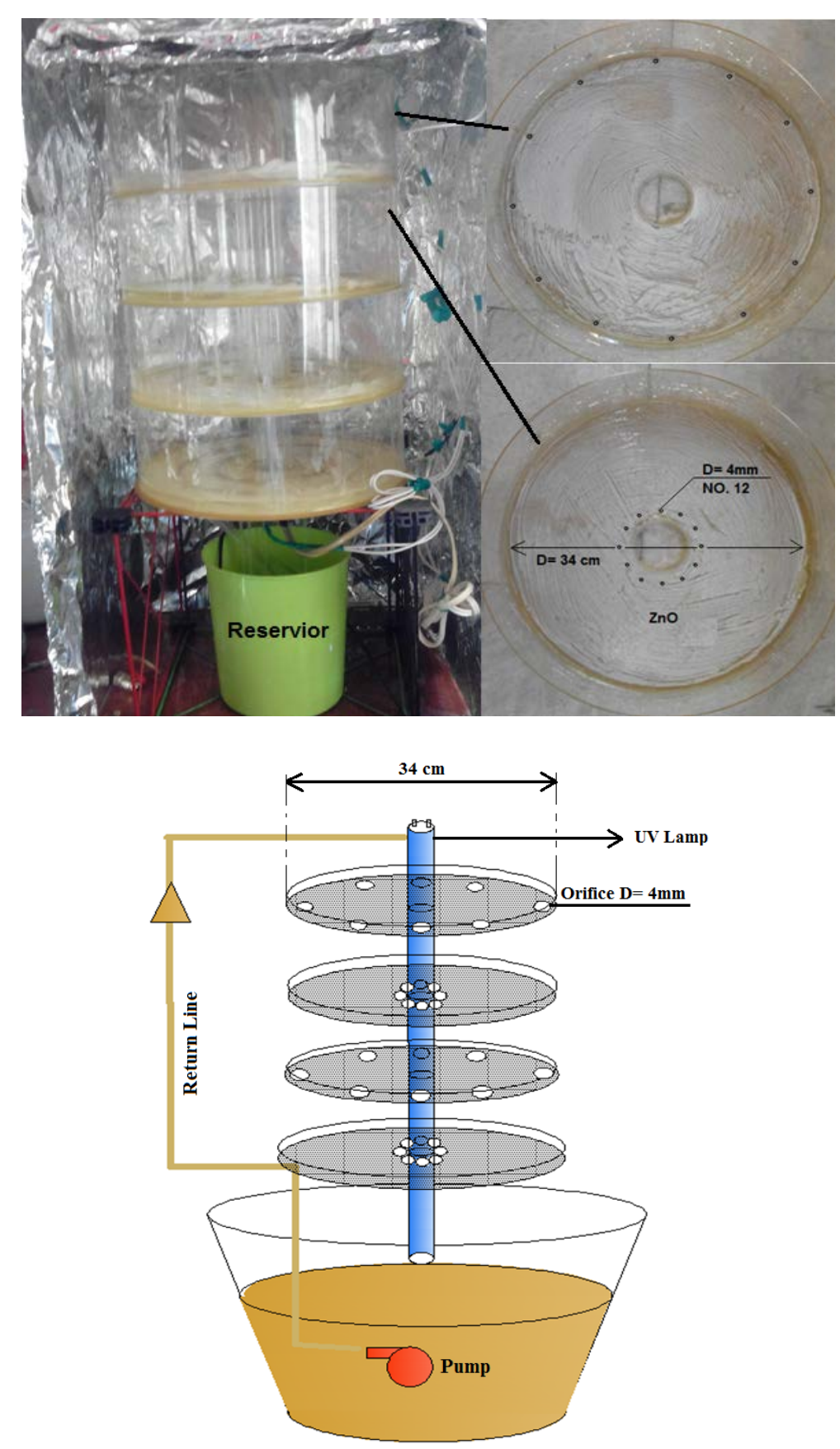

Figure 1. Schematic of the constructed photocatalytic reactor.

In this pilot, sewage flow is pumped to the highest level disc, and then it is transferred as cascade flow to lower level discs by small holes provided on each disc (12 small holes with $4 \mathrm{~mm}$ diameter). Also, this turbulence flow is spontaneously aerated. Therefore, mass transfer limitation in the reactors with immobilized bed is reduced significantly. A UVC $20 \mathrm{~W}$ lamp is located at the center of the reactor, and disc beds are coated by $\mathrm{ZnO}$ nanoparticles with area density of $20 \mathrm{gr} / \mathrm{m}^{2}$ [6]. In order to create artificial roughness, $5 \mathrm{~mm}$ baffles, as shown in Figure 2 have been used. These baffles are 


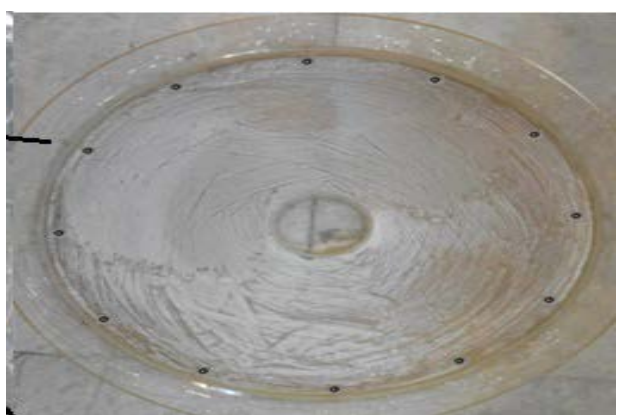

(a)

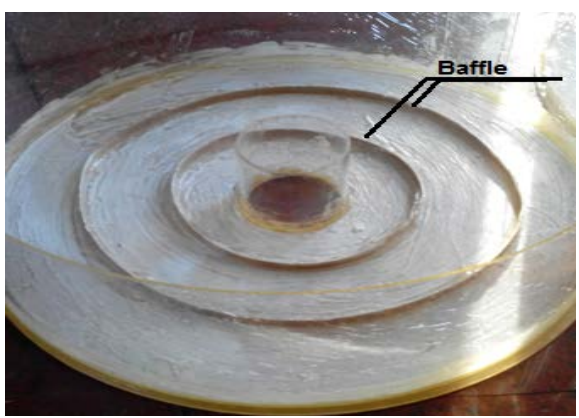

(b)

Figure 2. Disc of the reactor (a) without roughness (b) coated by artificial roughness.

placed at disc radiuses of 7 and $12 \mathrm{~cm}$. Again, sewage flow in storage supply is pumped to the highest level disc. Total volume of sewage treated in this treatment system is 5 liter which is restored with the flow rates of 40,60 and $80 \mathrm{cc} / \mathrm{sec}$.

\subsection{Materials and Equipment}

In this study, $\mathrm{ZnO}$ nanoparticles with purity of $99 \%$ (US research Nanomaterials Inc) are used. Sizes of particles vary from 10 to $90 \mathrm{~nm}$ and their surface area and density are $20-60 \mathrm{~m}^{2} / \mathrm{gr}$ and $5.606 \mathrm{gr} / \mathrm{cm}^{3}$, respectively (Figure 3).

Other materials including sodium hydroxide and sulfuric acid, made by The Merck Inc, are used for $\mathrm{pH}$ adjustment. Spectrophotometer (Hach Dr400) is utilized for determination of absorption ratio and dye concentration. Also, Fungilab Ultrasonic water bath and a digital weighting scale (PLS360-3-Kern) are employed for dispersing the algometry nanoparticles and material distribution, respectively. In this experiment, the pump is a submerged Soboti WP 3880 and the lamp is selected as a 20 W Philips (UVC).

\subsection{Numerical Model}

A two dimensional model is implemented by OpenFOAM software which is a finite volume based open-source computational fluid dynamic (CFD) package. Figure 4 depicts the geometry of the model that is a sector with diameter of $34 \mathrm{~cm}$ and the apex angle of $30 \mathrm{deg}$ ( $1 / 12$ of a complete disc). $5 \mathrm{cc} / \mathrm{sec}$ flow rate is considered for the model and non-slip condition for baffles and the model body is imposed. A $280 \times 30 \times 14$ mesh is defined for the domain at radial, angular and height directions as shown in

\section{Figure 4.}

\section{Results and Discussion}

\subsection{Effect of Initial Concentration on Photocatalytic Process}

Initial dye concentration is one of important parameter in photocatalytic process. Figure 5 shows the effect of various initial dye concentrations from 5 to $100 \mathrm{mg} / \mathrm{L}$ on photocatalytic process. The results shown in Figure 5 illustrates that an increase in the dye concentration will lower the removal rate. When the initial dye concentration in- 


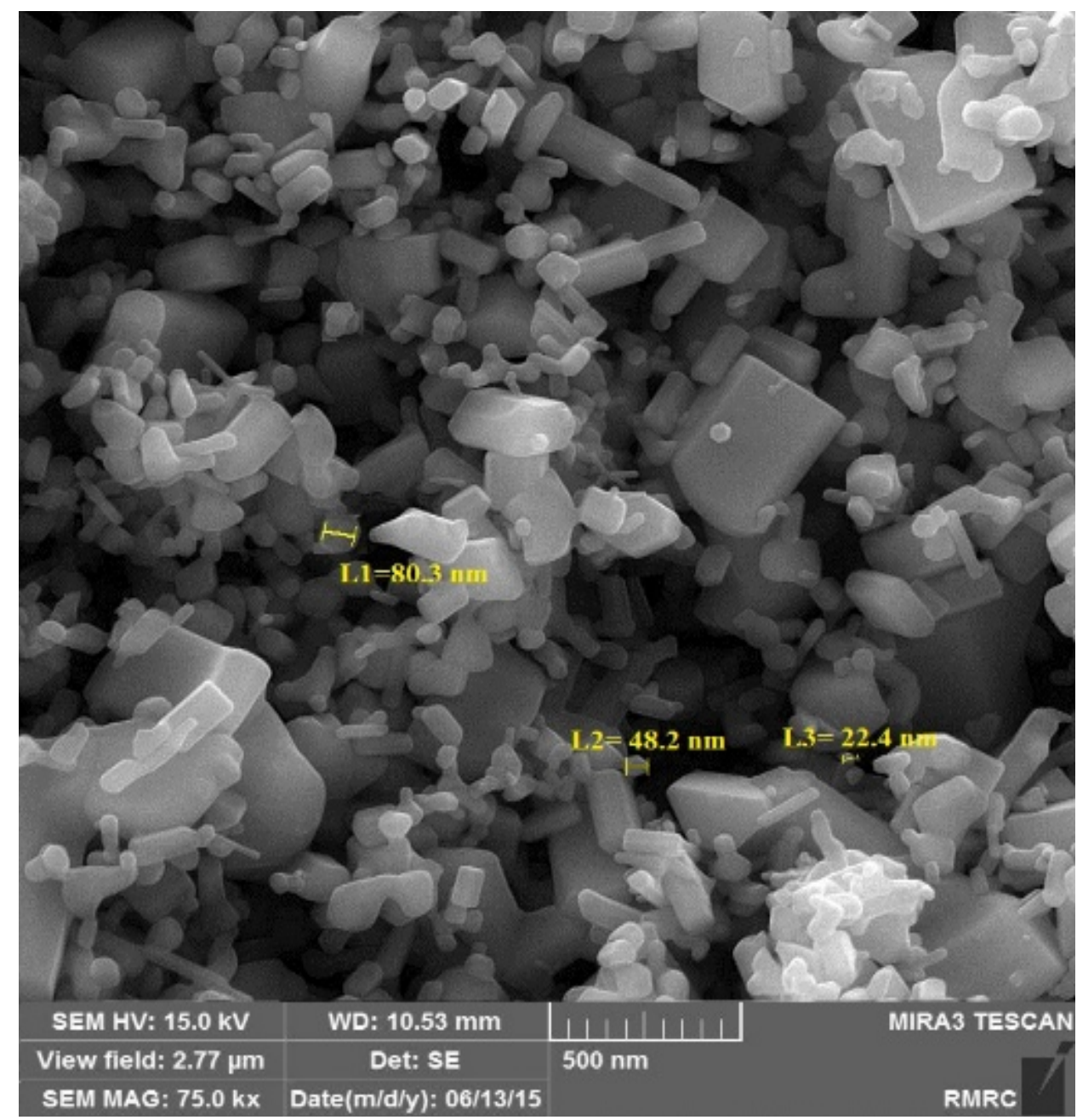

Figure 3. FESEM image of $\mathrm{ZnO}$ nanoparticles coated on surface.

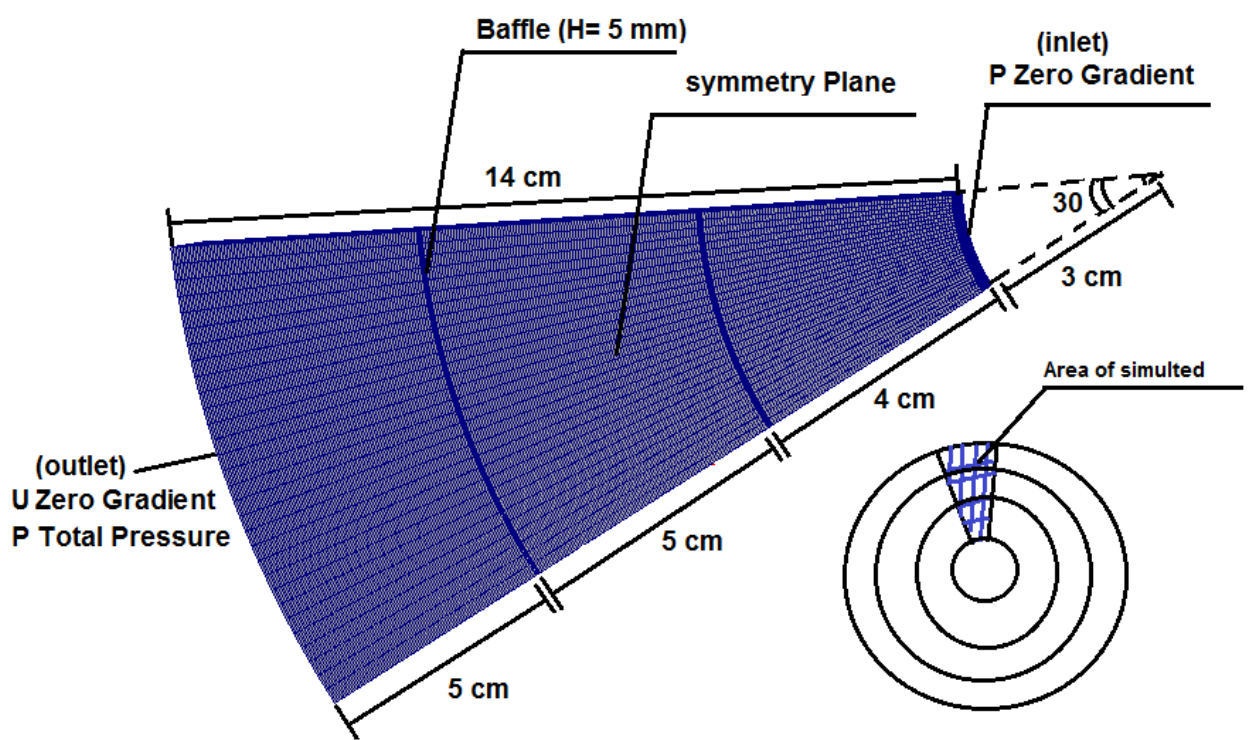

Figure 4. Domain of the numerical model and its generated grid. 


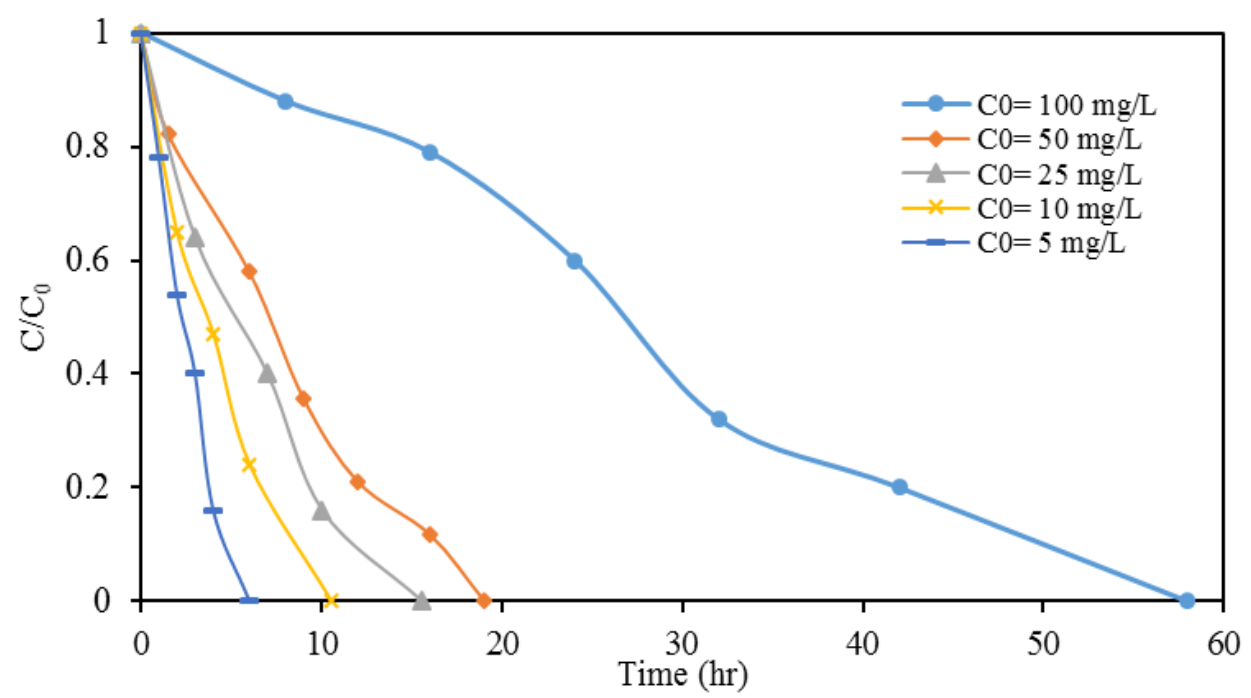

Figure 5. Effect of initial concentration on of photocatalytic decolorization of RY $81(\mathrm{Q}=40 \mathrm{cc} / \mathrm{s}$, without roughness, $\mathrm{pH}=7$ ).

creases from 5 to 100, the total time for complete degradation of RY81 increased from 6 to $57 \mathrm{hr}$. This affects the catalytic activity of $\mathrm{ZnO}$ nanoparticles and the number of active site on disk surface. Another impact of increasing dye concentration is related to changing of path length of photons entering the dye solution.

\subsection{Flow Rate Effects on Photocatalytic Process}

This research also evaluates the effects of flow rate on the RY81 removal. These experiments use dye concentrations of 10,50 and $100 \mathrm{mg} / \mathrm{L}$; and flow rates of 40, 60 and 80 cc/sec; $\mathrm{pH}$ of 7; and a $20 \mathrm{~W}$ UV lamp. In these experiments, Reynolds numbers are adjusted to be different. Figure 6 indicates the effect of flow rate, which varies from 40 to $80 \mathrm{cc} / \mathrm{sec}$, on the first order reaction rate in cascade disk reactor without roughness. Furthermore, final run-time for complete dye removal with different concentrations is reduced when flow rate is increased, Figure 6 indicates that flow rate increasing results in Reynolds number increasing and turbulence flow is formed. Probability of catalyst and pollution collision is increased and time of dye removal process is improved as turbulence effect enhances the collision [5]. This flow rate increasing (Reynolds number increasing) causes diffusion phenomenon to be happen between catalyst surface and dye molecules, and increases convection mass transfer rate [7].

\subsection{Effect of Artificial Relative Roughness on the Photocatalytic Process}

By coating roughness with height of $0.5 \mathrm{~cm}$ on the disc surface, total removal run-time is changed. Table 1 illustrates the effects of two parameters (relative roughness and flow rate). $10 \mathrm{mg} / \mathrm{L}$ dye removal subjected to flow rates of 40,60 and $80 \mathrm{cc} / \mathrm{sec}$ without considering roughness takes $10.5,9.5$, and 8.2 hours, respectively. These times are reduced to 10, 9 and 7.4 hours, respectively, when roughness is considered. 


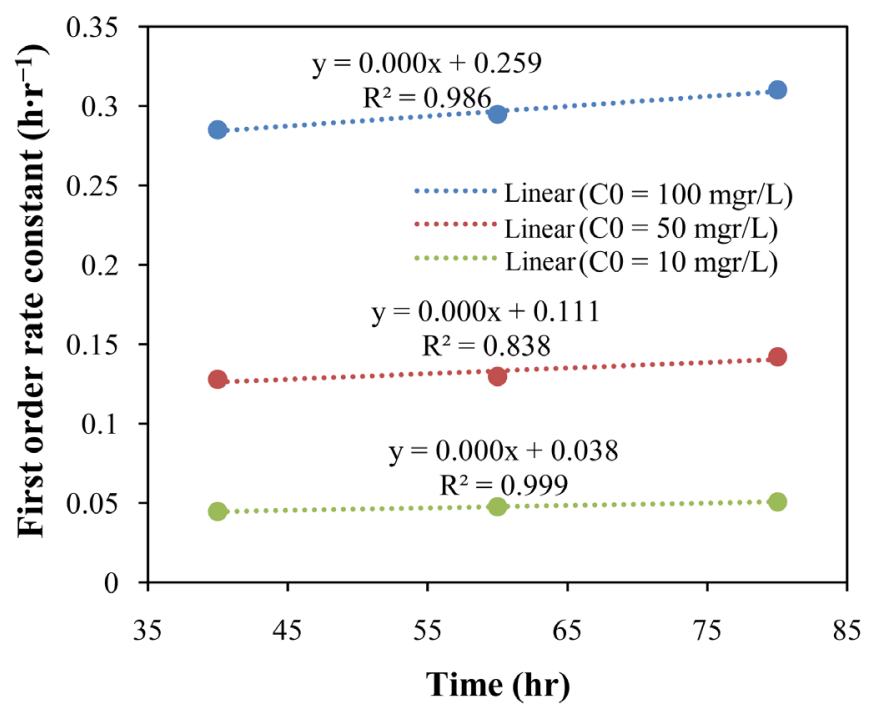

Figure 6. Effect of flow rate on the reaction rate of photocatalytic process without roughness.

Table 1. Total dye removal time of RY81 for initial concentrations and different flow rates with/ without roughness

\begin{tabular}{ccccc}
\hline C (mg/L) & Q (cc/s) & $\begin{array}{c}\text { Time for Destruction } \\
\text { of RY81 without Roughness }\end{array}$ & $\begin{array}{c}\text { Time for Destruction of } \\
\text { RY81 with Roughness }\end{array}$ & $\begin{array}{c}\text { Time Reduction } \\
(\%)\end{array}$ \\
\hline 10 & 40 & 10.5 & 10 & 4.8 \\
10 & 60 & 9.5 & 9 & 5.3 \\
10 & 80 & 8.2 & 7.7 & 6.1 \\
50 & 40 & 22 & 21 & 4.5 \\
50 & 60 & 20 & 18.6 & 7.0 \\
50 & 80 & 19 & 18 & 5.3 \\
100 & 40 & 57 & 54 & 5.3 \\
100 & 60 & 52 & 49 & 5.8 \\
100 & 80 & 50 & 47 & 6.0 \\
\hline
\end{tabular}

Therefore, total dye removal time is decreased about 5.6\%. Artificial roughness increases flow velocity of the spaces between baffles so that the velocity and Reynolds number become two times greater. Figure 7 indicates that as Reynolds number increase, overall dye removal time is decreased [8] [9]. Also, artificial roughness makes turbulence flow and the formed vortexes reduce dead space and increase mass transfer rate [10] [11]. As depicted in Figure 8, vortex is formed between two baffles, enhancing turbulence effects at the catalyst surface.

Figure 8 compares the effects of flow rates and relative roughness for pollution with concentration of $10 \mathrm{mg} / \mathrm{L}$. Overall removal time is 10.5 hours for flow rate of $40 \mathrm{cc} / \mathrm{sec}$ without roughness, while it is 7.7 hours for flow rate of $80 \mathrm{cc} / \mathrm{sec}$ when roughness is considered, which the time is reduced to $27 \%$.

Moreover, Figure 9 compares dye removal times for different flow rates with and without considering roughness. The result shows that increasing flow rates and existence of roughness lead to improve removal time. 


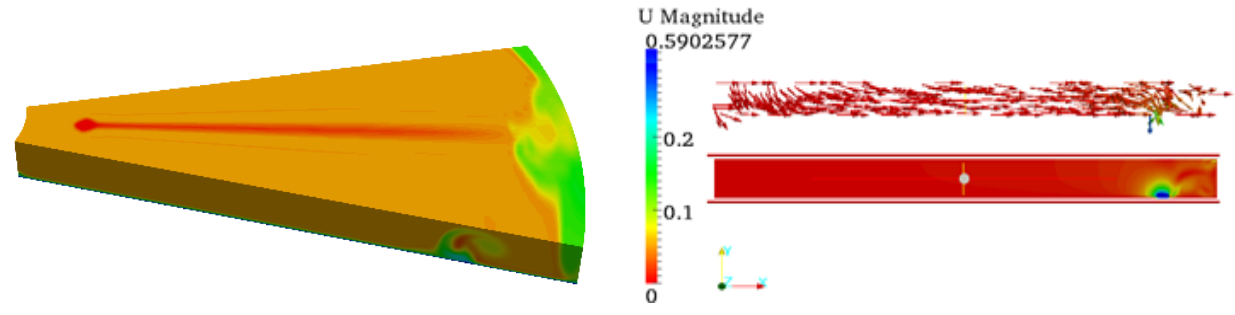

(a)

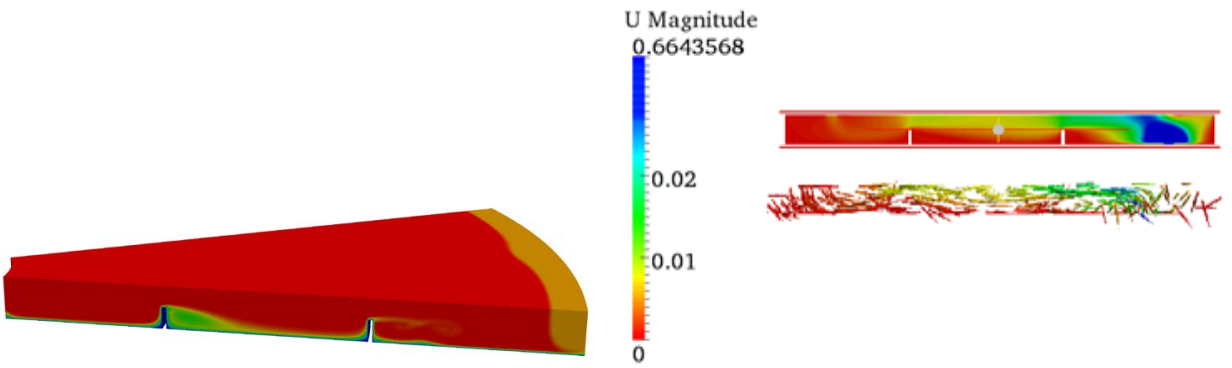

(b)

Figure 7. Velocity of hydraulic model of reactor: (a) without roughness; and (b) with roughness.

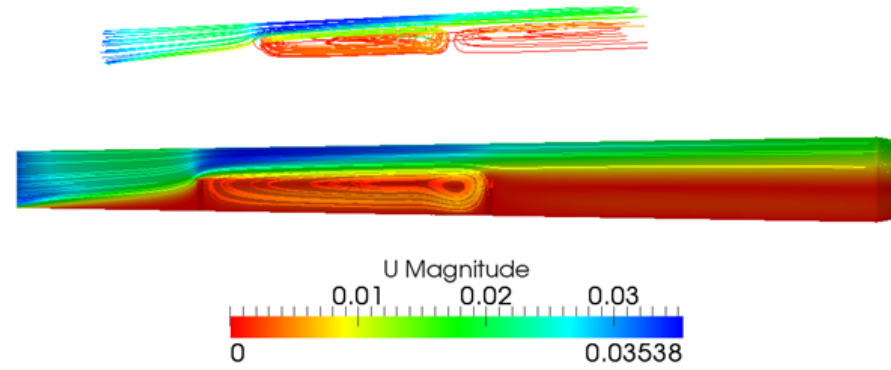

Figure 8. Vortex formed in the hydraulic model.

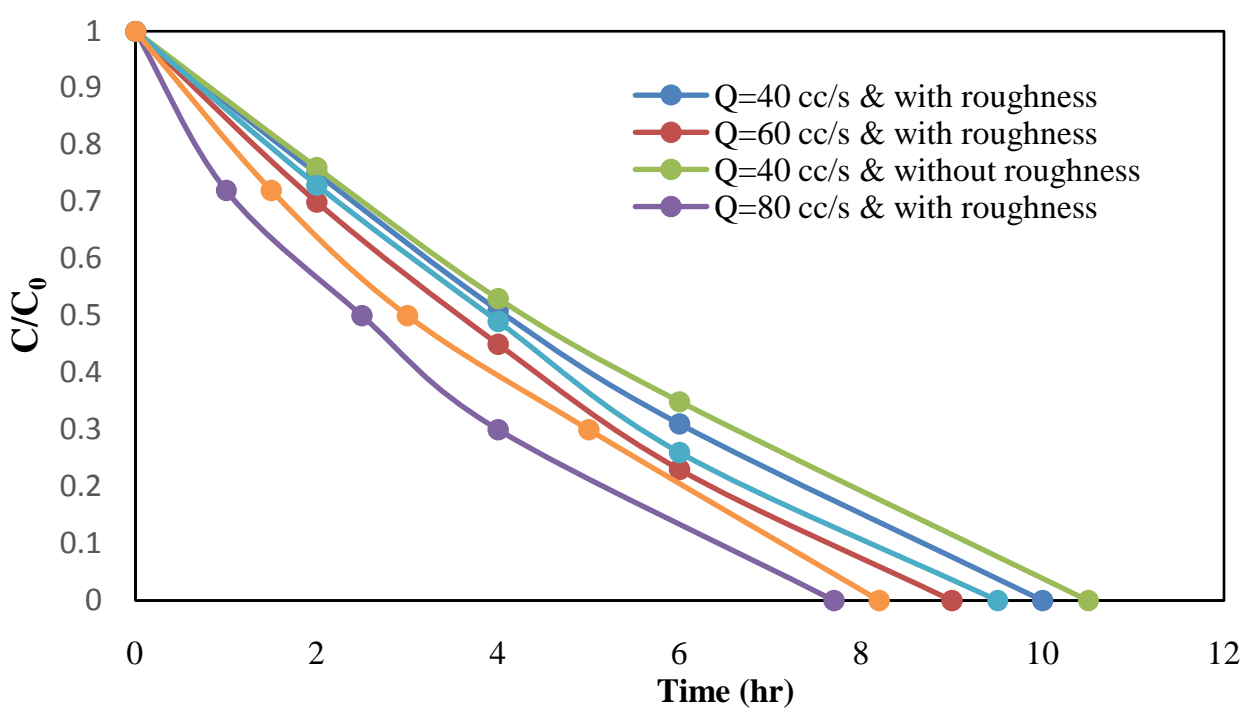

Figure 9. Effects of flow rates and artificial relative roughness on the RY81 dye removal with concentration of $10 \mathrm{mg} / \mathrm{L}$. 


\section{Conclusions}

Efficiency improvement of a reactor by non-mechanical equipment and roughness change due to using baffles is an economical way for sewage treatment and improvement of photocatalytic reaction rate. Mass transfer rate can also be increased by increasing flow rate leading to turbulence flow. A reactor with roughness property is more reliable and requires low maintenance cost.

Effects of relative artificial roughness are assessed in a cascade disc reactor by CFD simulation. The results demonstrate that the imposed in roughness, separate the boundary layer at downstream of each barrier and vortex is formed between barrier increasing vertical mixture of the flow. Furthermore, experimental results show that mass transfer phenomenon and photocatalytic rate process can be increased by changing hydraulic parameters such as flow rate and roughness. Because dead space is reduced by increasing the roughness, and Reynolds number and flow velocity becomes two times greater without spending energy. Hydraulic condition modifications increase the photocatalytic reaction rate to $26 \%$.

\section{References}

[1] Reddy, S.S., Kotaiah, B. and Reddy, N.S.P. (2008) Color Pollution Control in Textile Dyeing Industry Effluents Using Tannery Sludge Derived Activated Carbon. Bulletin of the Chemical Society of Ethiopia, 22.

[2] Basturk, E. and Karatas, M. (2015) Decolorization of Antraquinone dye Reactive Blue 181 Solution by UV/H2O2 Process. Journal of Photochemistry and Photobiology A: Chemistry, 299, 67-72. https://doi.org/10.1016/j.jphotochem.2014.11.003

[3] Mehrjouei, M., Müller, S. and Möller, D. (2015) A Review on Photocatalytic Ozonation Used for the Treatment of Water and Wastewater. Chemical Engineering Journal, 263, 209-219. https://doi.org/10.1016/j.cej.2014.10.112

[4] Zou, L., Li, Y. and Hu, E. (2005) Photocatalytic Decolorization of Lanasol Blue CE Dye Solution Using a Flat-Plate Reactor. Journal of Environmental Engineering, 131, 102-107. https://doi.org/10.1061/(ASCE)0733-9372(2005)131:1(102)

[5] Vezzoli, M., Martens, W.N. and Bell, J.M. (2011) Investigation of Phenol Degradation: True Reaction Kinetics on Fixed Film Titanium Dioxide Photocatalyst. Applied Catalysis A: General, 404, 155-163. https://doi.org/10.1016/j.apcata.2011.07.025

[6] Malayeri, H.Z., Ayati, B. and Ganjidoust, H. (2014) Photocatalytic Phenol Degradation by Immobilized Nano ZnO. Water Environment Research, 86, 771-778. https://doi.org/10.2175/106143014X13975035526301

[7] Yu, H., Zhang, K. and Rossi, C. (2007) Theoretical Study on Photocatalytic Oxidation of VOCs Using Nano-TiO 2 Photocatalyst. Journal of Photochemistry and Photobiology A: Chemistry, 188, 65-73. https://doi.org/10.1016/j.jphotochem.2006.11.021

[8] Zhang, Y., Stefanakos, E.K. and Goswami, D.Y. (2013) Effect of Photocatalytic Surface Roughness on Reactors Effectiveness for Indoor Air Cleaning. Building and Environment, 61, 188-196. https://doi.org/10.1016/j.buildenv.2012.12.018

[9] Kumar, J. and Bansal, A. (2013) Photocatalytic Degradation in Annular Reactor: Modelization and Optimization Using Computational Fluid Dynamics (CFD) and Response Surface Methodology (RSM). Journal of Environmental Chemical Engineering, 1, 398-405. 
https://doi.org/10.1016/j.jece.2013.06.002

[10] Ahmed, S., et al. (2011) CFD Simulation of Turbulence Promoters in a Water Treatment Reactor.

[11] Vezzoli, M. (2012) Intrinsic Kinetics of Titania Photocatalysis: Simplified Models for Their investigation.

Submit or recommend next manuscript to SCIRP and we will provide best service for you:

Accepting pre-submission inquiries through Email, Facebook, LinkedIn, Twitter, etc. A wide selection of journals (inclusive of 9 subjects, more than 200 journals)

Providing 24-hour high-quality service

User-friendly online submission system

Fair and swift peer-review system

Efficient typesetting and proofreading procedure

Display of the result of downloads and visits, as well as the number of cited articles Maximum dissemination of your research work

Submit your manuscript at: http://papersubmission.scirp.org/

Or contact msce@scirp.org 